\title{
Constraining the primordial spectrum of metric perturbations from gravitino and moduli production
}

\author{
Antonio L. Maroto* \\ Physics Department, Stanford University, Stanford, California 94305-4060
}

(Received 24 November 2001; published 29 March 2002)

\begin{abstract}
We consider the production of gravitinos and moduli fields from quantum vacuum fluctuations induced by the presence of scalar metric perturbations at the end of inflation. We obtain the corresponding occupation numbers, up to first order in perturbation theory, in terms of the power spectrum of the metric perturbations. We compute the limits imposed by nucleosynthesis on the spectral index $n_{s}$ for different models with constant $n_{s}$. The results show that, in certain cases, such limits can be as strong as $n_{s}<1.12$, which is more stringent than those coming from primordial black hole production.
\end{abstract}

DOI: 10.1103/PhysRevD.65.083508

PACS number(s): 98.80.Cq

\section{INTRODUCTION}

In the framework of inflationary cosmology, density perturbations are generated when metric and inflaton quantum fluctuations become super-Hubble sized during inflation and reenter the horizon as classical fluctuations in the radiation or matter dominated eras [1]. Typically, the scale corresponding to the present horizon size $\left(H_{0}^{-1} \sim 3000 \mathrm{Mpc}\right)$ left the horizon about $60 e$-folds before the end of inflation, and therefore one expects a primordial spectrum of metric perturbation spanning a range of scales from thousands of megaparsecs down to $e^{-60} / H_{0} \sim 10^{-23} \mathrm{Mpc}$. The current observations of cosmic microwave background anisotropies and large scale structure have started providing us with precise measurements of the primordial spectrum at large scales (1000-1 Mpc). Smaller scales are much more difficult to probe since their evolution has already become nonlinear or the corresponding fluctuations have been erased by various damping effects in the early universe. However, still there is a window open to those scales which is provided by primordial black holes. When a perturbation crosses inside the horizon with relative large amplitude $1 / 3 \leqslant \delta \leqslant 1$, the perturbed region stops expanding and starts collapsing eventually forming a black hole [2-4]. If such black holes do not evaporate fast enough they could overclose the universe and, therefore, limits on their primordial abundance are required. If they do evaporate by the present time, limits are still needed in order for the evaporation products do not disrupt primordial nucleosynthesis. Such limits can be translated into bounds on the power spectrum, which are particularly useful to constrain models of inflation in which perturbations grow at smaller scales, i.e., when the corresponding spectral index is larger than that of Harrison and Zeldovich $n_{s}=1$.

Apart from the generation of primordial black holes, metric perturbations at small scales can have other interesting effects, which we will explore in this paper. The presence of metric inhomogeneities is known to break the conformal flat-

\footnotetext{
* On leave of absence from Dept. Física Teórica, Universidad Complutense de Madrid, 28040, Madrid, Spain. Email address: maroto@itp.stanford.edu
}

ness of the Friedmann-Robertson-Walker (FRW) metric. In fact, such effect is more important as we move to smaller and smaller scales, and thus, in principle, one expects to find a strong deviation from conformal flatness right at the end of inflation, provided the smallest scales reenter the horizon with sufficiently large amplitude. However, we will find that even perturbations with very small amplitude can have important cosmological effects. One of the physical consequences of conformal noninvariance is the possibility of creating particles from vacuum fluctuations $[5,6]$. Recently $[7,8]$ this mechanism has been applied to the generation of large scale magnetic fields after inflation. Also in [9], the production of fermions from metric perturbations during preheating has been considered. Other sources for fermion production after inflation have been studied in [10]. In this paper we will be interested in the creation of a different kind of relics which can also have important consequences in cosmology, namely gravitinos and moduli fields.

In supergravity theories, the gravitino is the spin-3/2 superpartner of the graviton field. Its couplings to the rest of matter fields are typically suppressed by the Planck mass scale, which implies that gravitinos can live very long and even decay after nucleosynthesis destroying the nuclei created in this period. A similar effect is due to the presence of moduli fields, corresponding to the radii of extra dimensions in higher dimensional theories. This imposes very strong contraints on the primordial abundance of this kind of particles, thus typically we have $n / s<10^{-12}-10^{-14}$ for masses of the relics in the range $m=10^{3}-10^{2} \mathrm{GeV}[11-15]$. Traditionally, these constraints are translated into upper limits on the reheating temperature of the universe. In order to avoid their thermal overproduction we need $T_{R}<10^{8}-10^{9} \mathrm{GeV}$. More recently, non-thermal production of gravitinos and moduli during preheating has been considered [16-21]. In those works, it has been shown how the coherent oscillations of the inflaton field after inflation could produce a large amount of relics that could conflict in some cases with the above limits. In fact, in the case of moduli fields, the limits imposed on the reheating temperature can be as strong as $T_{R}<100 \mathrm{GeV}$, if the gravitational or the production during preheating are taken into account [20]. In this work, we will show how in certain models of inflation, metric perturbations 
can also give rise to a relevant amount of gravitinos and moduli. In those cases, the nucleosynthesis limits discussed above impose stringent constraints on the perturbations power spectrum.

The plan of the paper goes as follows. In Sec. II, we consider the gravitino equations of motion in an inhomogeneous background and obtain their perturbative solutions. In Sec. III, using the Bogolyubov technique, we compute the total number of gravitinos produced as a function of the power spectrum and compare the results with the nucleosynthesis bounds. Section IV is devoted to a similar analysis but using moduli fields. Section V contains the main conclusions of the work and finally we have summarized some useful formulas in the Appendix.

\section{GRAVITINO FIELD EQUATIONS IN INHOMOGENEOUS BACKGROUNDS}

The massless Rarita-Schwinger equation is conformally invariant, which implies that gravitinos are not produced from vacuum fluctuations in a FRW background. In the case of massive gravitinos in FRW, they can be produced either gravitationally [23] or due to the time dependence of the mass during preheating [16-18]. However, since in this work we are interested in the effect of metric perturbations, we will consider that the mass of the gravitino is constant and very small and effectively we can neglect it in the calculations. This is a good approximation for models in which the gravitino mass is smaller than the Hubble parameter after inflation, since gravitinos will be produced with energies much higher than their mass. For simplicity, we will also consider only the production of helicity $\pm 3 / 2$ particles which is technically simpler. The helicity $\pm 1 / 2$ production exhibits additional subtleties related to the fact that their equations of motion are only consistent if the gravitational background is a solution of the supergravity equations of motion. In order to get consistent inflationary supergravity models, one usually requires the introduction of several chiral superfields. In that case, the equations of motion for the helicity $\pm 1 / 2$ gravitino cannot be decoupled from those of the fermionic components of the superfields [21]. In addition, in the context of the production during preheating, if the inflationary sector is decoupled from the sector responsible for the present supersymmetry breaking, it is the fermionic partner of inflaton that is produced more abundantly rather than the longitudinal gravitino [22]. These points make the calculations more involved. However, in our case, we do not expect any new physical effects coming from helicity $\pm 1 / 2$, since on physical grounds, the production mechanism is purely gravitational and the helicity $\pm 1 / 2$ components will be produced with similar abundance to the helicity $\pm 3 / 2$ states.

Let us then consider the massless Rarita-Schwinger equation in an external gravitational background:

$$
\epsilon^{\mu \nu \rho \sigma} \gamma_{5} \gamma_{\nu} D_{\rho} \psi_{\sigma}=0
$$

where $\psi_{\sigma}$ is a Majorana spinor satisfying $\psi_{\sigma}=C \bar{\psi}_{\sigma}^{T}$ with $C$ $=i \hat{\gamma}^{2} \hat{\gamma}^{0}$ the charge conjugation matrix. The covariant derivative is given by

$$
D_{\mu} \psi_{\sigma}=\left(\partial_{\mu}+\Gamma_{\mu}\right) \psi_{\sigma}-\Gamma_{\mu \sigma}^{\lambda} \psi_{\lambda}
$$

and the spin connection by $\Gamma_{\mu}=-\frac{1}{8} \Gamma_{\mu}^{a b}\left[\hat{\gamma}_{a}, \hat{\gamma}_{b}\right]$. With these definitions, we have $D_{\mu} \gamma_{\nu}=0$ and because of the totally antisymmetric tensor, the Christoffel symbols do not contribute to Eq. (1). As usual, latin indices $a, b, \ldots$ refer to the tangent-space tensors, whereas greek ones $\mu, \nu, \ldots$ are used for curved background objects. The different gamma matrices are related by $\gamma_{\mu}=e_{\mu}^{a} \hat{\gamma}_{a}$, where the vierbein satisfies $e_{\mu}^{a} e_{\nu}^{b} g^{\mu \nu}=\eta^{a b}$, with $\eta_{a b}$ the Minkowski space metric. form:

For the background metric we will take the following

$$
g_{\mu \nu}=g_{\mu \nu}^{0}+h_{\mu \nu}
$$

where

$$
g_{\mu \nu}^{0} d x^{\mu} d x^{\nu}=a^{2}(\eta)\left(d \eta^{2}-\delta_{i j} d x^{i} d x^{j}\right)
$$

is the flat FRW metric in conformal time and

$$
h_{\mu \nu} d x^{\mu} d x^{\nu}=2 \Phi a^{2}(\eta)\left(d \eta^{2}+\delta_{i j} d x^{i} d x^{j}\right)
$$

is the most general form of the linearized scalar metric perturbation in the longitudinal gauge and where it has been assumed that the spatial part of the energy-momentum tensor is diagonal, as indeed happens in the inflationary or perfect fluid cosmologies [1]. In this expression $\Phi(\eta, \vec{x})$ is the gauge invariant gravitational potential.

Contracting Eq. (1) with $\gamma_{\lambda} \gamma_{\mu}$ we get

$$
2 i D_{\lambda}\left(\gamma^{\sigma} \psi_{\sigma}\right)-2 i \not D \psi_{\lambda}=0
$$

As commented before we are asumming that metric perturbations are classical perturbation which are produced during inflation. In this sense, it is a good approximation to consider that $\Phi$ vanishes asymptotically in the past. Since we are interested in the smallest scale perturbations, which are going to produce the leading effects, we will consider only those perturbations that reenter the horizon during the radiation era. In this case, once they reenter they start oscillating with damped amplitude. For that reason, we will also take $\Phi \rightarrow 0$ when $\eta \rightarrow \infty$. The vanishing of the perturbations allows us to define conformal vacuum states in the asymptotic regions. Thus, in those regions, the above equation reduces to a Dirac-like equation $i \not D^{(0)} \psi_{\mu}^{(0)}=0$ (see [16]) where the index (0) denotes the unperturbed object. The corresponding positive frequency solution with momentum $\vec{p}$ and helicity $\lambda= \pm 3 / 2$ can be written as:

$$
\begin{aligned}
\psi_{\mu}^{(0) p, \pm}(x) & =a^{-1 / 2}(\eta) \widetilde{\psi}_{\mu}^{(0) \pm}(\vec{p}, \eta) e^{i \vec{p} \vec{x}} \\
& =\frac{1}{\sqrt{2 p V a(\eta)}} u(\vec{p}, \pm) \epsilon_{\mu}(\vec{p}, \pm) e^{i \vec{p} \vec{x}-i p \eta}
\end{aligned}
$$

where the polarization vectors are given by 


$$
\begin{aligned}
\epsilon_{\mu}(\vec{p},+)= & \frac{1}{\sqrt{2}}(0, \cos \theta \cos \phi-i \sin \phi, \cos \theta \sin \phi \\
& +i \cos \phi,-\sin \theta) \\
\epsilon_{\mu}(\vec{p},-)= & -\frac{1}{\sqrt{2}}(0, \cos \theta \cos \phi+i \sin \phi, \cos \theta \sin \phi \\
& -i \cos \phi,-\sin \theta)
\end{aligned}
$$

with $p^{\mu}=(p, p \sin \theta \cos \phi, p \sin \theta \sin \phi, p \cos \theta)$ and the normalization $\quad \epsilon_{\mu}^{*}(\vec{p}, m) \epsilon^{\mu}(\vec{p}, n)=-\delta_{m n}, \quad p^{\mu} \epsilon_{\mu}(\vec{p}, m)$ $=p^{\mu} \epsilon_{\mu}^{*}(\vec{p}, m)=0$. The helicity $r, s= \pm 1 / 2$ spinors are chosen such that they satisfy: $u^{\dagger}(\vec{p}, r) u(\vec{p}, s)=2 p \delta_{r s}$. Here we are working in a finite box with comoving volume $V$ and we will take the infinite volume limit at the end of the calculations. Notice that the above solutions satisfy the additional constraints [16]:

$$
p^{\mu} \psi_{\mu}^{(0) p, \pm}(x)=0, \quad \gamma^{\mu} \psi_{\mu}^{(0) p, \pm}(x)=0 .
$$
form:

We will look for perturbative solutions of Eqs. (6) in the

$$
\psi_{\mu}=\psi_{\mu}^{(0) p, \lambda}+\psi_{\mu}^{(1)}+\cdots
$$

where, as mentioned above, $\psi_{\mu}^{(0) p, \lambda}$ is the solution of the unperturbed equation given in Eq. (7). Expanding Eq. (6) up to first order in the perturbation we find

$$
2 i D_{\mu}^{(0)}\left(\gamma^{(0) \sigma} \psi_{\sigma}^{(1)}\right)-2 i \not D^{(0)} \psi_{\mu}^{(1)}-2 i \not D^{(1)} \psi_{\mu}^{(0)}=0
$$

where we have used the constraint equation (10). In the Appendix we have given the expressions for the perturbative expansions of the different terms in the above equation. Since we are only interested in the evolution of those states which asymptotically (in the past and in the future) give rise to helicity $\pm 3 / 2$ gravitinos, we will project these equations along those helicity states. The projectors in the asymptotic regions are given in Fourier space by

$$
P_{ \pm 3 / 2}^{\mu}(\vec{k})=\epsilon^{\mu *}(\vec{k}, \pm) P_{ \pm 1 / 2}(\vec{k})
$$

where $P_{r}$ are helicity $r= \pm 1 / 2$ projectors satisfying $P_{r}(\vec{k}) u(\vec{k}, s)=u(\vec{k}, s) \delta_{r s}$. Let us assume for simplicity that the three-momentum is pointing along the $\mathrm{z}$-direction $k^{\mu}$ $=(|k|, 0,0, k)$. In this case, the projectors take a very simple form

$$
\begin{gathered}
\epsilon^{\mu *(\vec{k}, \pm)}=\frac{1}{\sqrt{2}}(0,1, \mp i, 0) \\
P_{ \pm 1 / 2}(\vec{k})=\frac{1}{2}\left(1 \pm \gamma^{5} \hat{\gamma}^{0} \hat{\gamma}^{3}\right) .
\end{gathered}
$$

Notice that the $\mu=0$ equation in Eq. (12) does not contribute to the projected equations. Using the fact that $P_{ \pm 3 / 2}^{\mu}(\vec{k}) k_{\mu} \psi$
$=0$ and $P_{ \pm 3 / 2}^{\mu}(\vec{k}) \gamma_{\mu} \psi=0$ for an arbitrary spinor $\psi$, we see that the first term in Eq. (12) vanishes when projected and we are left with

$$
P_{ \pm 3 / 2}^{i}(\vec{k}) \int \frac{d^{3} x}{(2 \pi)^{3 / 2}} e^{i \vec{k} \vec{x}}\left(\mathbb{D}^{(0)} \psi_{i}^{(1)}+\mathbb{D}^{(1)} \psi_{i}^{(0)}\right)=0 .
$$

Using the formulas in the Appendix we get the following expression for the equations of motion:

$$
\begin{aligned}
0= & \left(\hat{\gamma}^{0} \partial_{0}-i \hat{\gamma}^{j} k_{j}\right) \widetilde{\psi}_{ \pm 3 / 2}^{(1)}(\vec{k}, \eta) \\
& -P_{ \pm 3 / 2}^{i}(\vec{k})\left[\Phi\left(\hat{\gamma}^{0} \partial_{0}-i \hat{\gamma}^{j} p_{j}\right) \widetilde{\psi}_{i}^{(0) \lambda}(\vec{p}, \eta)\right] \\
& -\frac{i}{2} \Phi P_{ \pm 3 / 2}^{i}(\vec{k})\left[\left(k_{j}+p_{j}\right) \hat{\gamma}^{j} \widetilde{\psi}_{i}^{(0) \lambda}(\vec{p}, \eta)\right]
\end{aligned}
$$

where

$$
\widetilde{\psi}_{ \pm 3 / 2}^{(1)}(\vec{k}, \eta)=a^{1 / 2} P_{ \pm 3 / 2}^{i}(\vec{k}) \psi_{i}^{(1)}(\vec{k}, \eta)
$$

with $\psi_{i}^{(1)}(\vec{k}, \eta)$ the corresponding Fourier mode, defined as usual by $f(\vec{k}, \eta)=(2 \pi)^{-3 / 2} \int d^{3} x e^{i \vec{k} x} f(x)$. We have already defined $\widetilde{\psi}_{i}^{(0) \lambda}(\vec{p}, \eta)$ in Eq. (7). Finally we have denoted $\Phi(\vec{k}+\vec{p}, \eta)$ simply by $\Phi$

Notice that since $\widetilde{\psi}_{i}^{(0) \lambda}(\vec{p}, \eta)$ is a solution of the unperturbed equations of motion, the second term in the previous equation vanishes. Finally, in order to reduce Eq. (16) to a standard (inhomogeneous) harmonic oscillator equation, we multiply by $\left(\hat{\gamma}^{0} \partial_{0}-i \hat{\gamma}^{j} k_{j}\right)$, and we get:

$$
\left(\partial_{0}^{2}+k^{2}\right) \widetilde{\psi}_{ \pm 3 / 2}^{(1)}(\vec{k}, \eta)+J_{ \pm 3 / 2}=0 .
$$

The spinor current is given by:

$$
\begin{aligned}
J_{ \pm 3 / 2}= & \frac{1}{2}\left[\left(p^{2}+k^{2}-2 p k_{j} \hat{\gamma}^{0} \hat{\gamma}^{j}\right) \Phi\right. \\
& \left.-i \hat{\gamma}^{0}\left(k_{j} \hat{\gamma}^{j}-p \hat{\gamma}^{0}\right) \Phi^{\prime}\right] P_{ \pm 3 / 2}^{i}(\vec{k}) \widetilde{\psi}_{i}^{(0) \lambda}(\vec{p}, \eta)
\end{aligned}
$$

where the prime denotes a derivative with respect to conformal time $\eta$. Because of the presence of the inhomogeneous current, the initial positive frequency solution, with momentum $\vec{p}$ and helicity $\lambda$ in Eq. (7), will evolve into a linear superposition of positive and negative frequency modes, with different helicities and different momenta. Thus in the asymptotic future we find:

$$
\begin{aligned}
& \psi_{\mu}^{p, \lambda}(x) \underset{\eta \rightarrow \infty}{\longrightarrow} \sum_{\lambda^{\prime}= \pm} \sum_{k}\left(\alpha_{p k \lambda \lambda^{\prime}} \frac{u\left(\vec{k}, \lambda^{\prime}\right) \epsilon_{\mu}\left(\vec{k}, \lambda^{\prime}\right)}{\sqrt{2 k \operatorname{Va}(\eta)}} e^{i(\vec{k} \vec{x}-k \eta)}\right. \\
& \left.+\beta_{p k \lambda \lambda^{\prime}} \frac{u^{C}\left(\vec{k}, \lambda^{\prime}\right) \epsilon_{\mu}^{*}\left(\vec{k}, \lambda^{\prime}\right)}{\sqrt{2 k \operatorname{Va}(\eta)}} e^{-i(\vec{k} \vec{x}-k \eta)}\right)
\end{aligned}
$$

In order to obtain the Bogolyubov coefficients, we need to solve Eq. (18). Up to first order in perturbations, we have: 


$$
\begin{aligned}
\widetilde{\psi}_{ \pm 3 / 2}^{p, \lambda}(\vec{k}, \eta)= & \widetilde{\psi}_{ \pm 3 / 2}^{(0) p, \lambda}(\vec{k}, \eta) \\
& +\frac{1}{k} \int_{\eta_{0}}^{\eta} J_{ \pm 3 / 2} \sin \left(k\left(\eta-\eta^{\prime}\right)\right) d \eta^{\prime}
\end{aligned}
$$

where $\eta_{0}$ denotes the initial time in the remote past when the perturbations were switched off. Comparing this expression with the above expansion, it is very simple to get an explicit expression for the Bogolyubov coefficients [24]:

$$
\beta_{p k \lambda \mp}=-\frac{i}{2 k \sqrt{2 k V}} \int_{\eta_{0}}^{\eta_{1}} u^{C \dagger}(\vec{k}, \mp) J_{ \pm 3 / 2} e^{-i k \eta}
$$

where $\eta_{1}$ denotes the present time or any other instant in time after inflation in which the perturbations vanish again. Notice that the helicity projectors $P_{ \pm 3 / 2}^{\mu}$ project positive frequency modes on $\pm 3 / 2$ helicity states, whereas negative frequency modes are projected on $\mp 3 / 2$ states, which is why the $\beta$ coefficient subindexes are changed with respect to those of the current $J_{ \pm 3 / 2}$. The total number of gravitinos with comoving momentum $k$ is given by:

$$
N_{k}=\sum_{\lambda \lambda^{\prime}} \sum_{p}\left|\beta_{p k \lambda \lambda^{\prime}}\right|^{2}
$$

\section{GRAVITINO PRODUCTION FROM METRIC PERTURBATIONS}

As commented before, we will concentrate only on the effect of super-Hubble scalar perturbations whose evolution is relatively simple. For single-field inflationary scenarios we have [1]:

$$
\Phi(\vec{k}, \eta)=C_{k} \frac{1}{a} \frac{d}{d \eta}\left(\frac{1}{a} \int a^{2} d \eta\right)+D_{k} \frac{a^{\prime}}{a^{3}},
$$

the second term decreases during inflation and can soon be neglected. Thus, it will be useful to rewrite the perturbation as $\Phi(\vec{k}, \eta)=C_{k} \mathcal{F}(\eta)$. During inflation or preheating, superHubble perturbations evolve in time, whereas they are practically constant during radiation or matter eras. Notice that this expression is not appropriate in two-field models of inflation, since in that case super-Hubble gravitational fluctuations can be exponentially amplified during preheating [25] due to parametric resonance. by:

The power spectrum corresponding to Eq. (23) is given

$$
\mathcal{P}_{\Phi}(k)=\frac{k^{3}\left|C_{k}\right|^{2}}{2 \pi^{2} V}=A_{S}^{2}\left(\frac{k}{k_{C}}\right)^{n_{s}-1} .
$$

For simplicity we have assumed a power-law behavior with spectral index $n_{s}$ and we have set the normalization at the COBE scale $\lambda_{C} \simeq 3000 \mathrm{Mpc}$ with $A_{S} \simeq 5 \times 10^{-5}$. Although there are some models which predict this kind of behavior, in general we will have a dependence of the spectral index on the scale. However, in order to compare our results with those obtained from black holes, we will keep this simple form. In principle, there will be a maximum frequency cutoff $\kappa$ which corresponds to the perturbation with the smallest size produced during inflation. Typically this scale is roughly determined by the size of the horizon at the end of inflation $\kappa \sim a_{I} H_{I}$ where the $I$ index denotes the end of inflation (for a detailed discussion see [9]). We can obtain an explicit expression for the occupation number (22) in terms of the power spectrum. Taking the continuum limit $\Sigma_{p}$ $\rightarrow(2 \pi)^{-3 / 2} V \int d^{3} p$, we get:

$$
\begin{aligned}
N_{k}= & \sum_{\lambda \lambda^{\prime}} \int \frac{d p d \Omega}{(2 \pi)^{3 / 2}} p^{2} \cos ^{2}(\theta / 2) \\
& \times \frac{\left|C_{k+p}\right|^{2}}{4 k^{2} V}(p-k)^{2}\left|\int_{\eta_{0}}^{\eta_{1}}\left[i \mathcal{F}^{\prime}+\mathcal{F}(p-k)\right] d \eta\right|^{2}
\end{aligned}
$$

where we have used $k \eta \ll 1$ which is valid for super-Hubble modes and $\theta$ is the angle between $\vec{p}$ and the $z$ axis. The total comoving number density of gravitinos is given by $N$ $=\int d^{3} k N_{k}$. Typically this integral is dominated by the upper limit of integration, so that we can approximate $N \simeq \kappa^{3} N_{\kappa}$. Notice again that we are assuming no enhancement of highmomentum modes during preheating as it happens for instance in two-field models of inflation. It is then enough to calculate $N_{\kappa}$ :

$$
N_{\kappa} \simeq \frac{4 \pi^{3}}{(2 \pi)^{3 / 2}} \int d p p^{2} \frac{\mathcal{P}_{\Phi}(\kappa)}{\kappa^{3}} \simeq \frac{\sqrt{2 \pi^{3}}}{3} \mathcal{P}_{\Phi}(\kappa)
$$

Because of the Pauli exclusion principle $N_{k} \leqslant 1$, the violation of this bound signals the breakdown of the perturbative approach.

In order to get the ratio $n / s$ we need to calculate the entropy density at the end of inflation. If we assume that all the energy density in the inflaton field is instantaneously converted into radiation, we get a very high reheating temperature, which is already excluded by the thermal production of gravitinos as commented before. Therefore, for realistic models, reheating and thermalization should occur sufficiently late, so that we can have low reheating temperature. In that case, we must consider the stage of inflaton oscillations at the end of inflation. If the inflaton potential close to the minimum behaves as $V \sim \phi^{\alpha}$, then the energy density during oscillations scales as $\rho \sim a^{-6 \alpha /(\alpha+2)}$ [26]. Thus, although the physical number density scales as $n \sim a^{-3}$, the entropy density will scale as $s \simeq \rho^{3 / 4} \sim a^{-9 \alpha /[2(\alpha+2)]}$. This implies that during the oscillations and depending on the value of $\alpha$, the number density $n$ can be diluted with respect to the entropy density; it could remain constant or even increase. In the following, we will consider the usual potentials with $\alpha=2,4$ (notice that we are approximating the potential by simple powers only close to the minimum; during inflation their behavior can be completely different). For $\alpha=2$, the equation of state of the oscillating scalar field can be considered as that of non-relativistic particles; in this case $n / s$ decreases in time. For $\alpha=4$, the oscillations behave as 


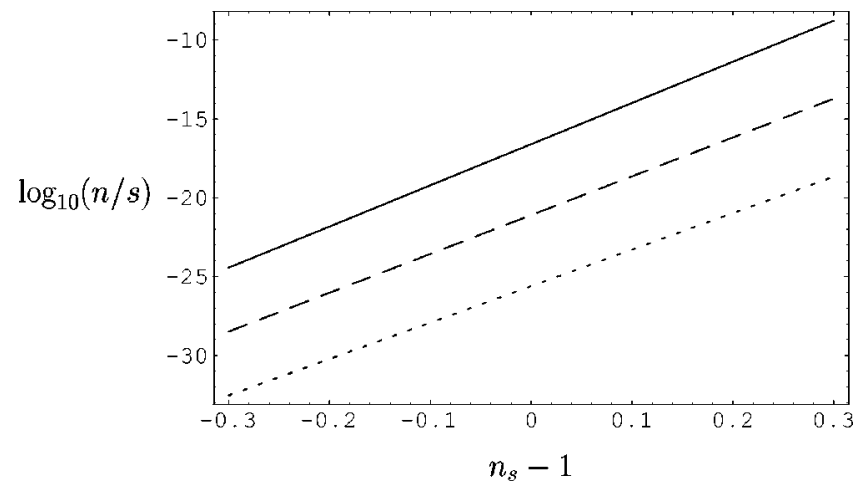

FIG. 1. Abundance of gravitinos $n / s$ versus spectral index for a model with $\alpha=4$ and $H_{I}=10^{13} \mathrm{GeV}$ (full line), $H_{I}=10^{10} \mathrm{GeV}$ (dashed line) and $H_{I}=10^{7} \mathrm{GeV}$ (dotted line). The results are independent of the reheating temperature.

radiation and $n / s$ is constant. Thus, taking this into account and setting $\kappa=a_{I} H_{I}$, we get for the ratio at the end of reheating:

$$
\frac{n}{s} \simeq \frac{N_{\kappa} H_{I}^{3}}{T_{R}^{3}}\left(\frac{a_{I}}{a_{R}}\right)^{3} \simeq N_{\kappa}\left(\frac{H_{I}}{\sqrt{3} M_{P}}\right)^{3 / 2}\left(\frac{T_{R}^{2}}{\sqrt{3} H_{I} M_{P}}\right)^{(4-\alpha) / 2 \alpha}
$$

where $M_{P}=(8 \pi G)^{-1 / 2}$. In order to compute $N_{\kappa}$ in the previous equation we need to know the value of the ratio $\kappa / k_{C}$. If the Hubble parameter remains constant during inflation we have $\kappa / k_{C}=a_{I} / a_{C}$, where $a_{C}$ denotes the scale factor at the moment when the scale $\lambda_{C}$ left the horizon. Therefore, in terms of the number of e-folds of inflation, we can write:

$$
\frac{\kappa}{k_{C}}=e^{N\left(k_{C}\right)}
$$

In the case $\alpha=2, N\left(k_{C}\right)$ is given by the well-known expression [27]:

$$
N\left(k_{C}\right)=53+\frac{1}{3} \ln \frac{T_{R}}{10^{10} \mathrm{GeV}}+\frac{2}{3} \ln \frac{V^{1 / 4}}{10^{14} \mathrm{GeV}}
$$

where $V$ is the value of the inflaton potential during inflation. A similar expression can be obtained for $\alpha=4$ :

$$
N\left(k_{C}\right)=56+\ln \frac{V^{1 / 4}}{10^{14} \mathrm{GeV}}
$$

which is independent of the reheating temperature. Substituting back in Eq. (27) and using Eqs. (24) and (26), we get:

$$
\begin{aligned}
\frac{n}{s} \simeq & \frac{\sqrt{2 \pi^{3}} A_{S}^{2}}{3}\left(\frac{H_{I}}{\sqrt{3} M_{P}}\right)^{3 / 2}\left(\frac{T_{R}^{2}}{\sqrt{3} H_{I} M_{P}}\right)^{(4-\alpha) / 2 \alpha} \\
& \times \exp \left(\left(n_{s}-1\right) N\left(k_{C}\right)\right) .
\end{aligned}
$$

In Fig. 1 we have plotted $n / s$ as a function of the spectral index for different models with $H_{I}=10^{13}, 10^{10}$ and $10^{7} \mathrm{GeV}$ with $\alpha=4$. In this case the results are independent of the

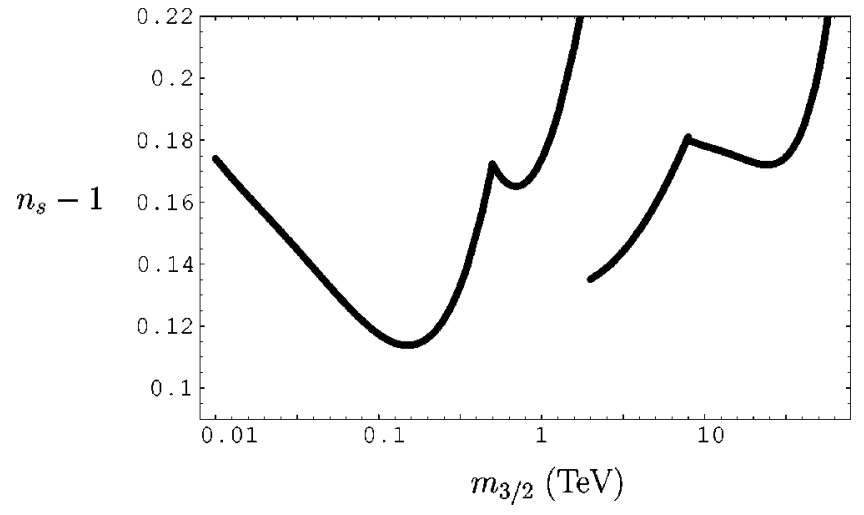

FIG. 2. Limits on the spectral index $n_{s}$ as a function of the gravitino mass $m_{3 / 2}$, from the generation of massive unstable gravitinos [11]. The right curve corresponds to the hadroproduction of D and ${ }^{4} \mathrm{He}[28]$, the left curve to the photofission of D and the photoproduction of D and ${ }^{3} \mathrm{He}$ [12]. The model of inflation has $\alpha=4$ and $H_{I}=10^{13} \mathrm{GeV}$.

reheating temperature. The nucleosynthesis bounds on the spectral index coming from the effects of the hadronic and radiative decays of gravitinos on elemental abundances are summarized in Fig. 2 for a typical model with $H_{I}$ $=10^{13} \mathrm{GeV}$. We see that the strongest bound comes from gravitino masses around $100 \mathrm{GeV}$ for which $n_{s}<1.12$. This limit improves that imposed by primordial black holes production. In fact, for $T_{R}<10^{9} \mathrm{GeV}$, such a limit comes from black holes which are evaporating today, and is given by $n_{s}<1.28[3,4]$. Notice that for $n_{s}=1.12$ and $\kappa / k_{C} \sim 10^{26}$ (which is the range of scales spanned in the model considered) we have $\mathcal{P}_{\Phi}(\kappa) \simeq 3 \times 10^{-6} \ll 1$, i.e., we are well inside the perturbative region. Notice that such perturbations are unable to create black holes, but still we have an important effect coming from the creation of relics.

In Fig. 3 we plot the number density versus spectral index for a model with $\alpha=2$ and $H_{I}=10^{13} \mathrm{GeV}$, for different values of the reheating temperature. Now because of the relative growth of entropy during the inflaton oscillations, the production is much weaker than in the previous case. In fact, in order to get a cosmologically relevant abundance with low reheating temperature, the spectral index should be larger than 1.4. However for those values we find $\mathcal{P}_{\Phi}>1$, i.e., we are out of the perturbative regime. For that reason for $\alpha=2$ we cannot obtain limits on the spectral index. In Fig. 3 we have also plotted the relative abundance for a model with high reheating temperature $\left(T_{R}=10^{15} \mathrm{GeV}\right)$ with a dashed line. The results show that even if we ignored the thermal production of gravitinos, we will find again the problem, but now coming from metric perurbations.

In [9] a weak production was obtained with numerical calculations for the $V=m^{2} \phi^{2} / 2$ and $V=\lambda \phi^{4} / 4$ models of chaotic inflation. This is due to the fact that those models predict a power spectra with negative tilt, i.e., $n_{s}<1$.

\section{MODULI PRODUCTION}

String theory and other higher-dimensional models include scalar fields that parametrize the shape of extra dimen- 


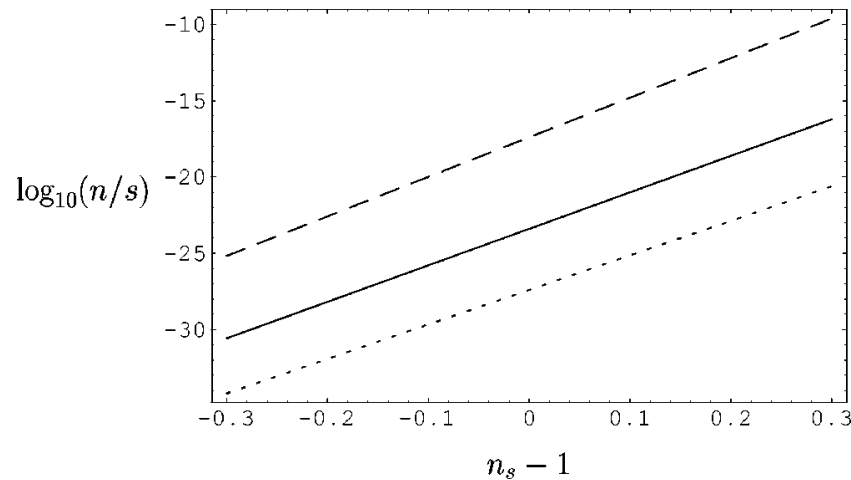

FIG. 3. Abundance of gravitinos $n_{3 / 2} / s$ versus spectral index $n_{s}$ for a model with $\alpha=2$ and reheating temperature $T_{R}=10^{9} \mathrm{GeV}$ (full line), $T_{R}=10^{15} \mathrm{GeV}$ (dashed line) and $T_{R}=10^{5} \mathrm{GeV}$ (dotted line), for $H_{I}=10^{13} \mathrm{GeV}$.

sions and whose couplings to matter fields are Planck mass suppressed. These kinds of fields can give rise to different cosmological problems [29]. First, if they were displaced from their minima in the early universe, we find the so-called classical moduli problem in which the energy density of the oscillations of the scalar fields behaves as non-relativistic matter, and can give relevant contribution to the energy density of the universe. If these fields decay during nucleosynthesis, we find a problem similar to that produced by gravitinos. The typical bounds on the primordial abundance of moduli are also similar, $n / s<10^{-12}-10^{-14}$ [11]. Second, moduli fields, can be produced from quantum fluctuations during inflation. In this case, the breaking of conformal invariance comes either from the minimal coupling to the scalar curvature or from their own mass. Thus the typical equation of motion for moduli is given by:

$$
\left(\square+m_{\chi}^{2}+\xi R\right) \chi=0 .
$$

In general, the moduli mass acquires corrections, coming from supersymmetry breaking effects during inflation or from non-renormalizable effects in the superpotential, thus one has $m_{\chi}^{2}=m^{2}+C^{2} H^{2}$, where $C$ is a model dependent parameter.

It has been shown [30] that the classical moduli problem is not present in the cases $C \ll 1$ or $C \gg 1$. The gravitational quantum production of moduli could also be avoided if conformal invariance is recovered, i.e., if $C=0, \xi=1 / 6$ and $m$ $=0$, although such fine tuning in the parameters seems to be difficult to obtain without invoking additional symmetries [18]. Since we are interested in the effect of metric perturbations on the moduli production, we will consider in the following that they are the only source of conformal symmetry breaking. In that case the above equation of motion reduces to:

$$
\left(\frac{1}{\sqrt{g}} \partial_{\mu} g^{\mu \nu} \sqrt{g} \partial_{\nu}+\frac{1}{6} R\right) \chi=0 .
$$

Using the form of the metric in Eq. (3) and the formulas in the Appendix, we find the following form of the linearized equation of motion:

$$
\tilde{\chi}^{\prime \prime}-\partial_{i} \partial_{i} \tilde{\chi}-4 \Phi^{\prime} \tilde{\chi}^{\prime}-\frac{1}{3}\left(\partial_{i} \partial_{i} \Phi\right) \tilde{\chi}-\Phi^{\prime \prime} \tilde{\chi}=0
$$

where $\tilde{\chi}=a \chi$. Again we look for solutions in the form $\tilde{\chi}$ $=\tilde{\chi}^{(0)}+\tilde{\chi}^{(1)}+\ldots$ where

$$
\tilde{\chi}^{(0)}=\frac{1}{\sqrt{2 p V}} e^{i(\vec{p} \vec{x}-p \eta)}
$$

corresponding to the initial conformal vacuum state. Fourier transforming the equation, we find for the first order correction:

$$
\tilde{\chi}^{(1)^{\prime \prime}}(\vec{k}, \eta)+k^{2} \tilde{\chi}^{(1)}(\vec{k}, \eta)+J=0
$$

where

$$
J=\frac{1}{\sqrt{2 p V}}\left(4 i p \Phi^{\prime}+\frac{1}{3}(\vec{p}+\vec{k})^{2} \Phi-\Phi^{\prime \prime}\right) e^{-i p \eta} .
$$

Using the analogous result to the gravitino case, we find for the Bogolyubov coefficients:

$$
\beta_{p k}=-\frac{i}{\sqrt{2 k V}} \int_{\eta_{0}}^{\eta_{1}} J e^{-i k \eta} d \eta
$$

and finally for the occupation number we get:

$$
\begin{aligned}
N_{k}= & \int \frac{d^{3} p}{(2 \pi)^{3 / 2}} \frac{\left|C_{p+k}\right|^{2}}{4 k p V} \\
& \times\left|\int_{\eta_{0}}^{\eta_{1}}\left(4 i p \mathcal{F}^{\prime}+\frac{1}{3}(\vec{p}+\vec{k})^{2} \mathcal{F}-\mathcal{F}^{\prime \prime}\right) d \eta\right|^{2}
\end{aligned}
$$

Assuming again that the total number density is dominated by those modes with $k \sim \kappa$, we have:

$$
\begin{aligned}
N_{\kappa} \simeq & \frac{32 \pi^{3}}{(2 \pi)^{3 / 2} \kappa} \int d p \frac{p^{3}}{\kappa^{3}} \mathcal{P}_{\Phi}(\kappa) \\
& \times\left|\int_{\eta_{0}}^{\eta_{1}}\left(i \mathcal{F}^{\prime}+\frac{\kappa^{2}}{12 p} \mathcal{F}-\frac{\mathcal{F}^{\prime \prime}}{4 p}\right) d \eta\right|^{2} \\
\simeq & \frac{(2 \pi)^{3 / 2}}{72} \mathcal{P}_{\Phi}(\kappa)
\end{aligned}
$$

where the time integral has been estimated to be $\simeq \kappa /(12 p)$, since $\mathcal{F} \simeq \mathcal{O}(1)$.

The ratio $n / s$ can be calculated in a similar fashion using Eq. (27) and the expression we have just obtained for $N_{\kappa}$. The results are plotted in Figs. 4 and 5 for different models with $\alpha=2,4$. In the case in which there is no relative dilution of $n$ with respect to $s$, we obtain that for a moderate value of the nucleosynthesis bound $n / s<10^{-13}$, the spectral index 


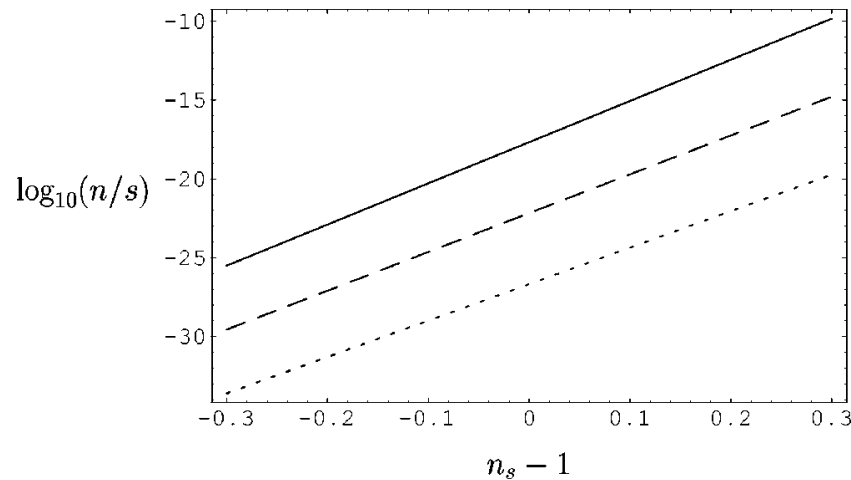

FIG. 4. Abundance of moduli $n_{\chi} / s$ versus spectral index $n_{s}$ for a model with $\alpha=4$ and $H_{I}=10^{13} \mathrm{GeV}$ (full line), $H_{I}=10^{10} \mathrm{GeV}$ (dashed line) and $H_{I}=10^{7} \mathrm{GeV}$ (dotted line). The results are independent of the reheating temperature.

should be $n_{s}<1.18$, whereas for the strongest one $n / s$ $<10^{-14}$, we get $n_{s}<1.14$ for $H_{I} \sim 10^{13} \mathrm{GeV}$. These limits are slightly weaker than those coming from gravitinos, in part due to the absence of spin degrees of freedom in this case. For $\alpha=2$ the limits are much less stringent as expected.

\section{CONCLUSIONS}

In this paper we have studied the production of helicity $\pm 3 / 2$ gravitinos and moduli fields from metric perturbations after inflation. The results show that those metric perturbations with very small wavelengths reentering the horizon right at the end of inflation induce a strong deviation from conformal flatness which is responsible for the production of a non-negligible amount of relics. In particular, if the power spectrum has a positive tilt $n_{s}>1$, as predicted by some models of hybrid inflation [31], the production could conflict with the limits imposed by nucleosynthesis. This in turn allows us to set stringent constraints on the spectral index in the particular case in which $n_{s}$ does not depend on the scale. When the energy density in the inflaton oscillations scales as radiation, the limits that we obtain $n_{s}<1.12$ are stronger than those coming from primordial black hole production. In the

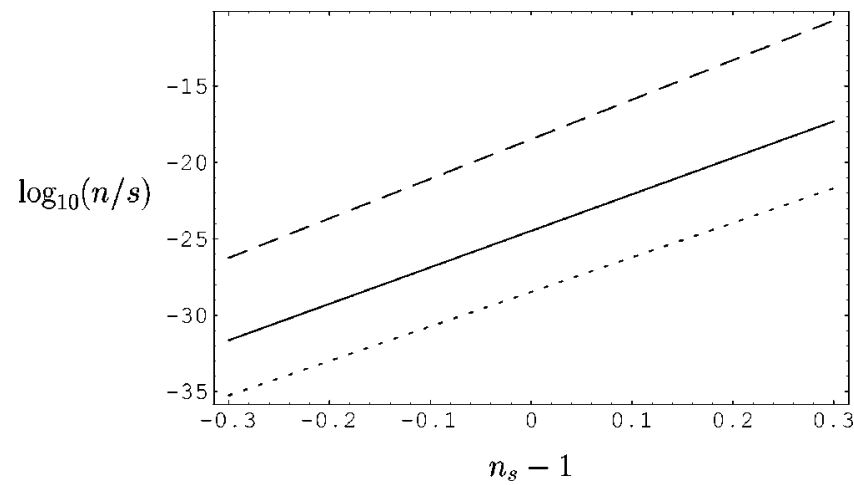

FIG. 5. Abundance of moduli $n_{\chi} / s$ versus spectral index $n_{s}$ for a model with $\alpha=2$ and reheating temperature $T_{R}=10^{9} \mathrm{GeV}$ (full line), $T_{R}=10^{15} \mathrm{GeV}$ (dashed line) and $T_{R}=10^{5} \mathrm{GeV}$ (dotted line), for $H_{I}=10^{13} \mathrm{GeV}$. case in which the energy in oscillations scales as nonrelativistic matter, the bounds are much weaker. When the spectral index depends on the scale, it is possible to translate the nucleosynthesis bounds into limits on the power spectrum at the smallest scale $\mathcal{P}_{\Phi}(\kappa)$. Using the results in the previous sections, we obtain $\mathcal{P}_{\Phi}(\kappa)<10^{-6}$, in models of inflation with $H_{I} \sim 10^{13} \mathrm{GeV}$ and $\alpha=4$. In conclusion, we consider that the production of gravitinos and moduli fields provides us with a new tool which can be useful to study the region of very small wavelengths in the primordial spectrum of metric peturbations.

\section{ACKNOWLEDGMENTS}

I am grateful to A. Linde and L. Kofman for useful comments. This work has been partially supported by the CICYT (Spain) projects AEN97-1693 and FPA2000-0956. The author also acknowledges financial support from the Universidad Complutense del Amo program.

\section{APPENDIX: USEFUL FORMULAS}

In this appendix we show some useful results for the perturbative expansions of different geometrical objects (see also [6]).

\section{Vierbein}

The vierbein expansion corresponding to the metric tensor in Eq. (3) is given by

$$
e_{\mu}^{b}=a\left(\eta_{\mu}^{b}+\frac{1}{2} h_{\mu}^{b}\right)
$$

where

$$
h_{0}^{0}=2 \Phi, \quad h_{j}^{i}=-2 \Phi \delta_{j}^{j} .
$$

\section{Gamma matrices}

The curved gamma matrices can be expanded as

$$
\gamma_{\mu}=e_{\mu}^{a} \hat{\gamma}_{a}=\gamma_{\mu}^{(0)}+\gamma_{\mu}^{(1)}+\cdots
$$

where

$$
\gamma_{0}^{(1)}=a \Phi \hat{\gamma}_{0}=\Phi \gamma_{0}^{(0)}, \quad \gamma_{i}^{(1)}=-a \Phi \hat{\gamma}_{i}=-\Phi \gamma_{i}^{(0)}
$$

\section{Spin connection}

For the spin connection appearing in the fermionic derivatives we find

$$
\begin{gathered}
\Gamma_{\mu}=\Gamma_{\mu}^{(0)}+\Gamma_{\mu}^{(1)}+\ldots \\
\Gamma_{\mu}^{(0)}=\frac{a_{, \lambda}}{4 a} \eta^{b \lambda} \eta_{\mu}^{a}\left[\hat{\gamma}_{a}, \hat{\gamma}_{b}\right]
\end{gathered}
$$




$$
\begin{aligned}
& \Gamma_{0}^{(0)}=0, \quad \Gamma_{j}^{(0)}=\frac{a^{\prime}}{2 a^{3}} \gamma_{j}^{(0)} \gamma_{0}^{(0)} \\
& \Gamma_{\mu}^{(1)}=\frac{1}{8}\left[\hat{\gamma}_{a}, \hat{\gamma}_{b}\right]\left(h_{\mu}^{a, b}+\eta_{\mu}^{b} h^{a \lambda} \frac{a_{, \lambda}}{a}+\eta^{b \lambda} h_{\mu}^{a} \frac{a_{, \lambda}}{a}\right) \\
& \Gamma_{0}^{(1)}=\frac{1}{2} \gamma_{0}^{(0)} \gamma^{(0) j} \Phi_{, j} \\
& \Gamma_{j}^{(1)}=-\frac{1}{4} \Phi_{, \mu}\left[\gamma_{j}^{(0)}, \gamma^{(0) \mu}\right]+\Phi \frac{a^{\prime}}{a^{3}} \gamma_{0}^{(0)} \gamma_{j}^{(0)} .
\end{aligned}
$$

\section{Christoffel symbols}

The non-vanishing Christoffel symbols for the metric (3) are given by

$$
\begin{aligned}
\Gamma_{\mu \nu}^{\lambda} & =\Gamma_{\mu \nu}^{(0) \lambda}+\Gamma_{\mu \nu}^{(1) \lambda}+\ldots \\
\Gamma_{00}^{(0) 0} & =\frac{a^{\prime}}{a}, \quad \Gamma_{i j}^{(0) 0}=\frac{a^{\prime}}{a} \delta_{i j}, \\
\Gamma_{0 j}^{(0) i} & =\frac{a^{\prime}}{a} \delta_{j}^{i} \\
\Gamma_{00}^{(1) 0} & =\Phi^{\prime}, \quad \Gamma_{0 i}^{(1) 0}=\Phi_{, i} \\
\Gamma_{i j}^{(1) 0} & =\left(-4 \frac{a^{\prime}}{a} \Phi^{\prime}-\Phi^{\prime}\right) \delta_{i j}, \\
\Gamma_{0 j}^{(1) i} & =-\Phi^{\prime} \delta_{j}^{i}
\end{aligned}
$$

$$
\begin{aligned}
\Gamma_{j k}^{(1) i} & =-\left(\Phi_{, k} \delta_{j}^{i}+\Phi_{, j} \delta_{k}^{i}-\Phi_{, l} \delta_{j k} \delta^{i l}\right) \\
\Gamma_{00}^{(1) i} & =\Phi_{, i} .
\end{aligned}
$$

\section{Scalar curvature}

Finally, for the scalar curvature we find up to first order:

$$
R=\frac{1}{a^{3}}\left[6 a^{\prime \prime}-12 \Phi a^{\prime \prime}-24 a^{\prime} \Phi^{\prime}+2 a \partial_{i} \partial_{i} \Phi-6 \Phi^{\prime \prime} a\right] .
$$

\section{Spinors}

The normalized spinors with helicity $\pm 1 / 2$ and momentum $\vec{p}$ that we have used in the text are:

$$
\begin{gathered}
u(\vec{p}, \pm)=\sqrt{p}\left(\begin{array}{c}
\chi_{ \pm} \\
\pm \chi_{ \pm}
\end{array}\right), \\
\chi_{+}=\left(\begin{array}{c}
e^{-i \phi / 2} \cos \left(\frac{\theta}{2}\right) \\
e^{i \phi / 2} \sin \left(\frac{\theta}{2}\right)
\end{array}\right), \\
\chi_{-}=\left(\begin{array}{c}
-e^{-i \phi / 2} \sin \left(\frac{\theta}{2}\right) \\
e^{i \phi / 2} \cos \left(\frac{\theta}{2}\right)
\end{array}\right) .
\end{gathered}
$$

[1] V.F. Mukhanov, H.A. Feldman, and R.H. Brandenberger, Phys. Rep. 215, 203 (1992).

[2] B.J. Carr, J.H. Gilbert, and J.E. Lidsey, Phys. Rev. D 50, 4853 (1994)

[3] A.M. Green and A.R. Liddle, Phys. Rev. D 56, 6166 (1997).

[4] A.M. Green, Phys. Rev. D 60, 063516 (1999).

[5] Ya.B. Zeldovich and A.A. Starobinsky, Sov. Phys. JETP 34, 1159 (1972).

[6] J.A. Frieman, Phys. Rev. D 39, 389 (1989); J. Céspedes and E. Verdaguer, ibid. 41, 1022 (1990); A. Campos and E. Verdaguer, ibid. 45, 4428 (1992).

[7] A.L. Maroto, Phys. Rev. D 64, 083006 (2001).

[8] B.A. Bassett, G. Pollifrone, S. Tsujikawa, and F. Viniegra, Phys. Rev. D 63, 103515 (2001).

[9] B.A. Bassett, M. Peloso, L. Sorbo, and S. Tsujikawa, Nucl. Phys. B622, 393 (2002).

[10] J. Baacke, K. Heitmann, and C. Patzold, Phys. Rev. D 58, 125013 (1998); P.B. Greene and L. Kofman, Phys. Lett. B 448, 6 (1999); A.L. Maroto and A. Mazumdar, Phys. Rev. D 59, 083510 (1999).

[11] J. Ellis, J.E. Kim, and D.V. Nanopoulos, Phys. Lett. 145B, 181 (1984).
[12] J. Ellis, G.B. Gelmini, J.L. López, D.V. Nanopoulos, and S. Sarkar, Nucl. Phys. B373, 399 (1992).

[13] J. Ellis, A. Linde, and D. Nanopoulos, Phys. Lett. 118B, 59 (1982).

[14] S. Sarkar, Rep. Prog. Phys. 59, 1493 (1996).

[15] M. Kawasaki and T. Moroi, Prog. Theor. Phys. 93, 879 (1995); T. Moroi, Ph.D. thesis, Tohoko University, 1995, hep-ph/9503210.

[16] A.L. Maroto and A. Mazumdar, Phys. Rev. Lett. 84, 1655 (2000).

[17] R. Kallosh, L. Kofman, A.D. Linde, and A. Van Proeyen, Phys. Rev. D 61, 103503 (2000).

[18] G.F. Giudice, I. Tkachev, and A. Riotto, J. High Energy Phys. 08, 009 (1999).

[19] A.L. Maroto and J.R. Peláez, Phys. Rev. D 62, 023518 (2000).

[20] A.S. Goncharov, A.D. Linde, and M.I. Vysotsky, Phys. Lett. 147B, 279 (1984); G.N. Felder, L. Kofman, and A.D. Linde, J. High Energy Phys. 02, 027 (2000); G.F. Giudice, A. Riotto, and I.I. Tkachev, ibid. 06, 020 (2001).

[21] R. Kallosh, L. Kofman, A.D. Linde, and A. Van Proeyen, Class. Quantum Grav. 17, 4269 (2000).

[22] H.P. Nilles, M. Peloso, and L. Sorbo, Phys. Rev. Lett. 87, 
051302 (2001); J. High Energy Phys. 04, 004 (2001).

[23] M. Lemoine, Phys. Rev. D 60, 103522 (1999).

[24] N.D. Birrell and P.C.W. Davies, Quantum Fields in Curved Space (Cambridge University Press, Cambridge, England, 1982).

[25] B.A. Bassett and F. Viniegra, Phys. Rev. D 62, 043507 (2000); F. Finelli and R. Brandenberger, ibid. 62, 083502 (2000).

[26] M.S. Turner, Phys. Rev. D 28, 1243 (1983).

[27] E.W. Kolb and M.S. Turner, The Early Universe (Addison-
Wesley, New York, 1990).

[28] M.H. Reno and D. Seckel, Phys. Rev. D 37, 3441 (1988).

[29] B. de Carlos, J.A. Casas, F. Quevedo, and E. Roulet, Phys. Lett. B 318, 447 (1993); T. Banks, D. Kaplan, and A. Nelson, Phys. Rev. D 49, 779 (1994).

[30] A.D. Linde, Phys. Rev. D 53, 4129 (1996).

[31] A.D. Linde, Phys. Rev. D 49, 748 (1994); J. García-Bellido, A.D. Linde, and D. Wands, ibid. 54, 6040 (1996). 